\title{
CLOVES syndrome caused by mosaic mutation in the PIK3CA gene identified in fibroblasts
}

\author{
Magdalena Kłaniewska', Małgorzata Rydzanicz², Joanna Kosińska², Mateusz Biela', \\ Anna Walczak², Elżbieta Szmida'1, Anna Rozensztrauch'1, Rafał Płoski², Robert Śmigiel' \\ 'Wroclaw Medical University, Poland \\ ${ }^{2}$ Medical University of Warsaw, Poland
}

\section{ABSTRACT}

CLOVES syndrome is a rare dysmorphic syndrome with multiple defects caused by somatic activating mutations in the PIK3CA gene on chromosome 3q26.32. There are currently less than 200 individuals worldwide living with CLOVES syndrome (OMIM: 612918, ORPHA: 140944). Due to the extremely low prevalence rate of CLOVES syndrome, few epidemiological data are available in the literature.

We report 4-year-old girl with somatic mutation in the PIK3CA gene (c.1357G>A) in fibroblast, revealed in the WES study, confirming the diagnosis of CLOVES syndrome.

CLOVES syndrome can be very difficult to diagnose, not only because of its extreme rarity, but also due to symptoms which vary both in range of symptoms and severity. Therefore, the case described by us may be helpful in the correct diagnosis of this rare disease in subsequent cases and makes an important contribution in rare disease diagnostics.

KEY WORDS:

mosaicism, CLOVES syndrome, PIK3CA mutation, overgrowth.

\section{INTRODUCTION}

For many years, overgrowth syndromes connected with vascular anomalies have been a challenge to diagnose. Recently, a separate group of patients has been identified with congenital lipomatous overgrowth, mixed vascular malformations, and dysregulated fat deposits [1]. The acronym CLOVE was first described by Sapp et al. in 2007 as congenital lipomatous (CL) overgrowth (O), vascular malformations (V), and epidermal nevi (E) [2]. Then Alomari in 2009 extended CLOVE to CLOVES to emphasize the association with scoliosis or skeletal and spinal anomalies and central nervous system malformations [3]. There are currently less than 200 individuals worldwide living with CLOVES syndrome (OMIM: 612918, ORPHA: 140944). The prevalence of the disease has been estimated at $<1 / 1,000,000$.
We present a case report of young patient with COLVES syndrome, and we point to the difficulties in diagnosis of a child with overgrowth and asymmetrical dysmorphic features including head, brain, face, subcutaneous and fat tissue, as well as skin.

\section{CASE REPORT}

A 4-year-old girl, born as in the $38^{\text {th }}$ week of pregnancy via caesarean section because of macrosomia. The birth weight was $4300 \mathrm{~g}$, length $59 \mathrm{~cm}$, head circumference $42 \mathrm{~cm}$, and chest circumference $34 \mathrm{~cm}$. Apgar score was 9 points at the $1^{\text {st }}$ minute. The pregnancy was uneventful. Family history was unencumbered.

Physical examination after birth revealed macrocephaly, facial dysmorphic features with asymmetry, and a congenital haemangioma. Ultrasound of the abdomen

\section{ADDRESS FOR CORRESPONDENCE:}

Magdalena Kłaniewska, Department of Pediatria and Rare Disorders, Wroclaw Medical University, 50-368

Wroclaw, Poland, e-mail: magdazdzie@gmail.com 


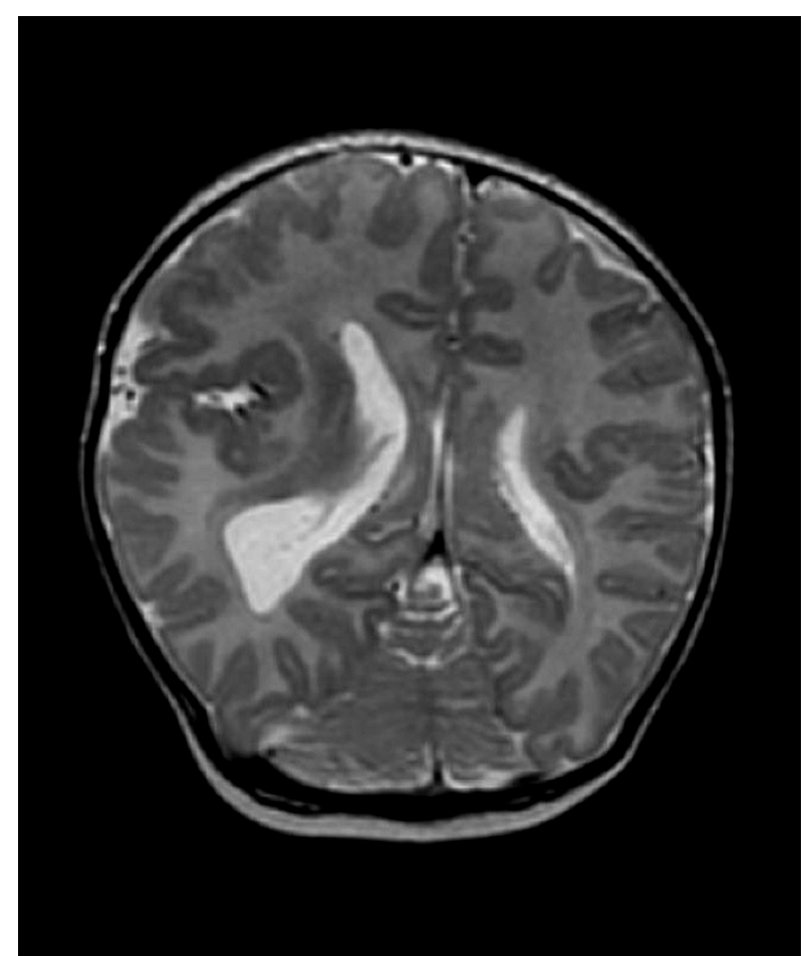

FIGURE 1. MRI in two weeks old patient shows hemimegaencephalia, schizencephalia, gray matter heterotopy and asymmetry of brain structures

and heart were normal. Cranial ultrasound scan and nuclear magnetic resonance, performed during the first days of life, revealed hemimegalencephaly, schizencephaly, and grey matter heterotopy (Fig. 1). In addition, gastroesophageal reflux disease (GERD) was diagnosed. The girl was initially suspected of Klippel-Trenaunay syndrome. Electroencephalography was abnormal but without any specific presentation. Infections from the TORCH group were excluded.

The girl was consulted by a clinical geneticist at the age of one year. Physical examination revealed low muscle tone, facial dysmorphic features with a leading sign of macrocephaly, and severe facial asymmetry including: eyelid fissures, cheeks, nose and mouth (Fig. 2). Extensive flat haemangiomas were observed mainly on the right side of the body. In addition, the child presented asymmetry of the lower and the upper limbs, especially asymmetry of the thigh and feet resulting from haemangiomas, as well as unidentified subcutaneous and fat tissue mass (Fig. 3). Other observed features were described: enlarged abdomen circumference (without liver and spleen enlargement), narrowed chest, and scoliosis. Moreover, macrodactyly of the third finger and a wide sandal gap were noticed. As a cause of these symptoms a mutation in the PIK3CA gene was suspected.

\section{INVESTIGATIONS}

Our patient firstly underwent targeted examination of the PIK3CA gene in chosen exons $(2,6,8,9,20)$ by

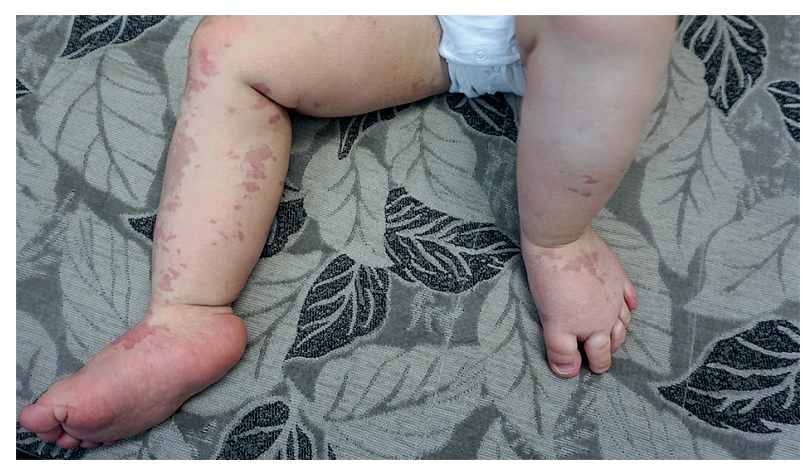

FIGURE 3. Asymmetry of the lower limbs with flat hemangiomas

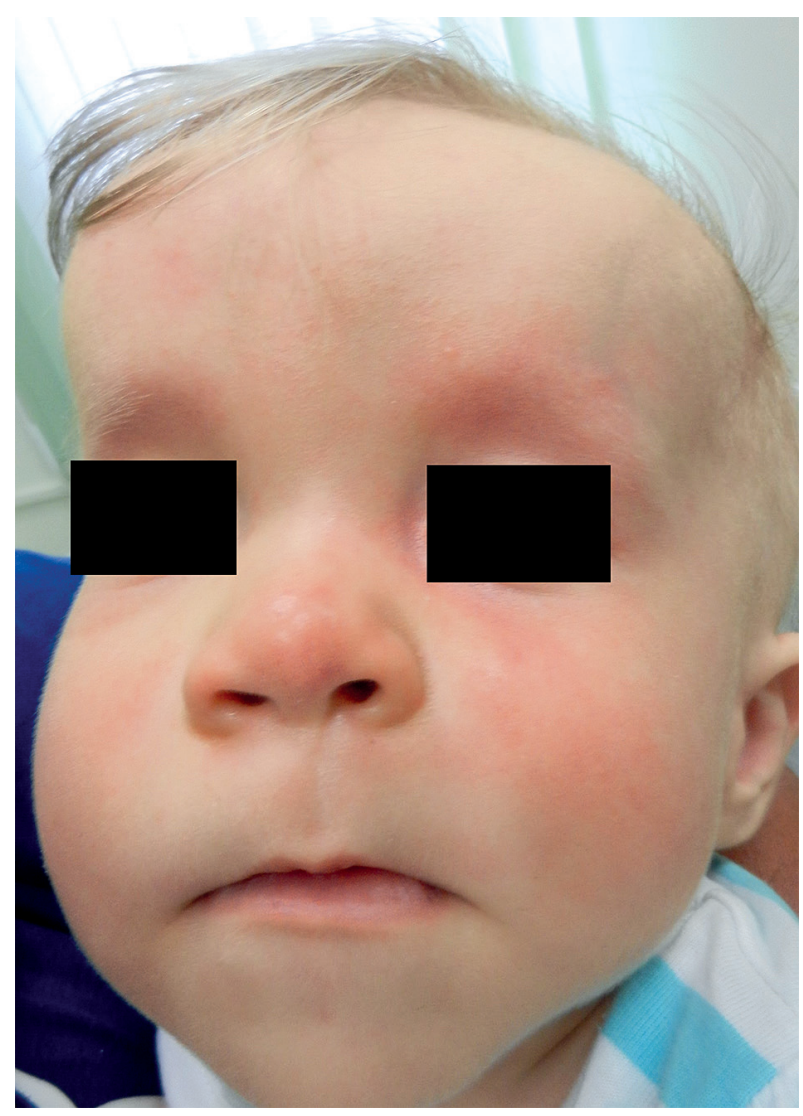

FIGURE 2. Facial dysmorphic features in 12 old month patient: macrocephaly, severe facial asymmetry

sanger sequencing from peripheral blood lymphocytes, which showed no pathogenic variants. For further examination, proband's DNA purified from skin-derived fibroblast was subjected into whole exome sequencing (WES). A library was prepared using SureSelect Human All Exon v5 kit (Agilent, Santa Clara, CA, USA) and paired-end sequenced $(2 \times 100 \mathrm{bp})$ on HiSeq 1500 (Illumina, San Diego, CA, USA). Bioinformatics analysis of raw WES data and variant prioritization were performed as previously described (PMID: 30254215). Selected variants were validated in proband's fibroblasts and studied in proband's blood as well as in blood collected from all available family members by amplicon deep sequencing performed using Nextera XT Kit (Illumina) and sequenced on a HiSeq 1500 (Illumina). 


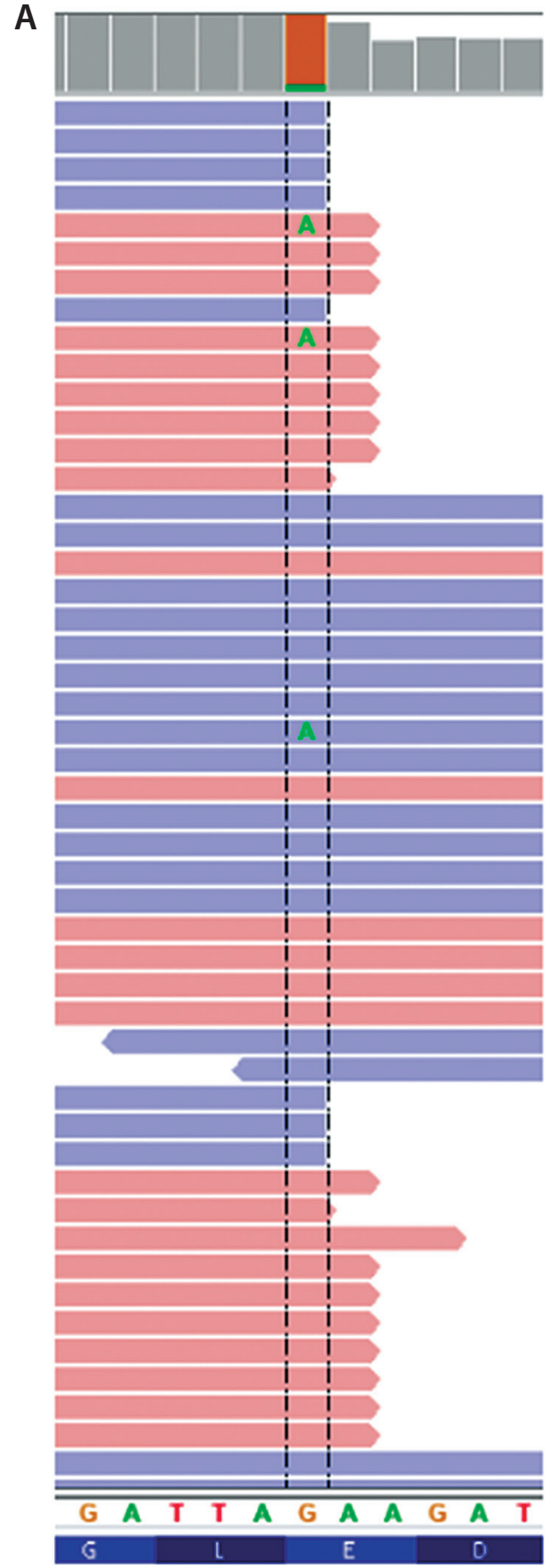

PIK3CA
B
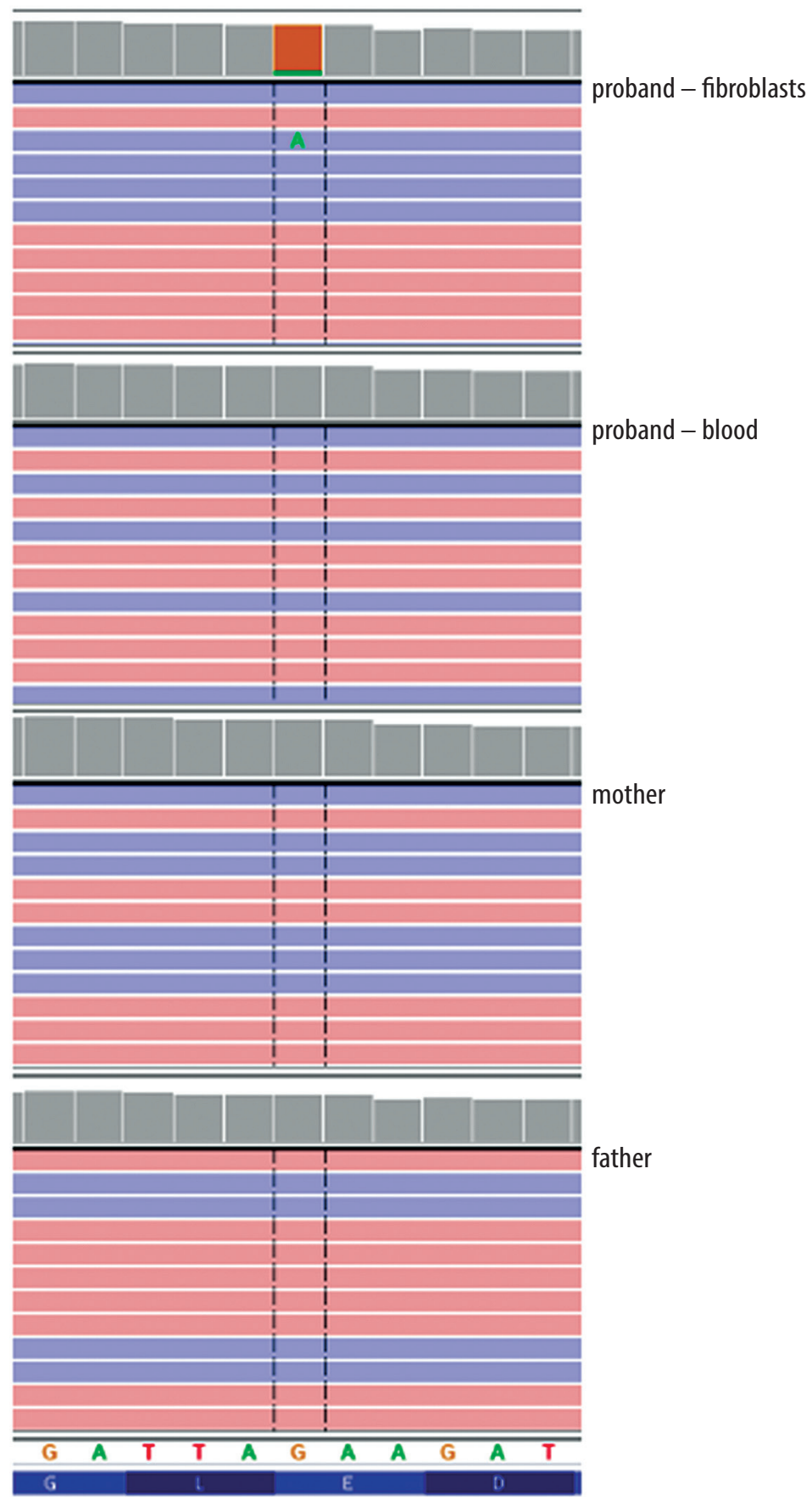

PIK3CA

FIGURE 4. Results of genetic study: A - missense variant p.(Glu453Lys) in PIK3CA gene identified in the proband's skin-derived fibroblasts by whole exome sequencing (total count $74, A-9 \%, G-91 \%$ ) and B - verified by amplicon deep sequencing in the proband's skin-derived fibroblasts (total count 1670, A - 10\%, G90\%), proband's blood (total count 20417, A - 0\%, G - 100\%), proband's mother (total count 556, $A-0 \%, G-100 \%$ ) and father (total count 7567, A - 0\%, G - 100\%). Integrative Genomic Viewer screen shots

Considering the proband's phenotype we prioritized a somatic missense heterozygous variant in the PIK3CA gene (hg19; chr3:g.178928079G >A, NM_006218.4:c.1357G>A, p.(Glu453Lys) (Fig. 4A). The variant was absent in all tested databases, including our in-house database of $>1000$ Polish exomes. In the ClinVar database p.(Glu453Lys) variant is described as probably pathogenic (https://www.ncbi.nlm.nih.gov/ clinvar/variation/376470/). The presence of p.(Glu453Lys) was excluded in the proband's parents (Fig. 4B).

\section{DISCUSSION}

CLOVES has been related to somatic activating mutations in the PIK3CA gene on chromosome 3q26.32 [4]. Somatic mutations appear during embryogenesis, resulting 
in two or more genetically separate cell lines. When the mutation influences cell signalling pathways changes in skin appearance can occur, occasionally with regional hyperplasia or even tumour susceptibility. The phenotype depends on the extent to which the mutation affects cellular function as well as on the number and organization of abnormal cells relative to normal cells [5]. PIK3CA is an upstream regulator of the Akt-mTOR cell-signalling pathway. This is balanced by the down-regulating activity of tensin homologue protein and the phosphatase [1].

The key feature of the CLOVES syndrome is a truncal lipomatous mass of variable size noted after birth [6]. Manifestations of overgrowth include asymmetric overgrowth with splaying of the feet and palmar or plantar overgrowth. Skeletal anomalies in the form of progressive scoliosis can occur [7]. Anomalies also affect feet and hands. Most commonly they appear in the form of wide feet and hands, macrodactyly typically involving the third finger or third toe, and a wide sandal gap [8]. Abnormalities seen occasionally include postaxial polydactyly and pectus excavatum [7]. Low- and high-flow vascular malformations belong to the major component of CLOVES syndrome, as well. Venous malformations, in the form of central, thoracic, and limb phlebectasia, have frequently been reported.

Central nervous system manifestations include hemimegalencephaly, polymicrogyria, a 4-layered cortex, abnormalities of the gray and white matter, ventriculomegaly dysgenesis of the corpus callosum, neuronal migration defects leading to seizures, tethered spinal cord, and neural tube defects [9]. Patients with CLOVES syndrome have various degrees of intellectual disability. Almost all patients with CLOVES syndrome are cognitively normal, but one report by Alomari revealed that neurological disorders were observed in about $50 \%$ of the study cohort [10]. CLOVES syndrome can be very tricky to diagnose, not only because of its extreme rarity, but also because of symptoms that vary in both presentation and severity.

The mutation indicated in our patient's tests has been described in the literature as associated with the PIK3CA-Related Overgrowth Spectrum (PROS). The term was created in 2013 by the National Institute of Health in Bethesda, to designate all the phenotypes caused by PIK3CA mutation. The phenotypes that contain PROS are as follows: megalencephaly, capillary malformation, polymicrogyria syndrome (MCAP), congenital lipomatous, overgrowth, vascular malformations, epidermal nevi, scoliosis (CLOVES), dysplastic megalencephaly (DMEG), fibroadipose hyperplasia or overgrowth (FAO), and macrodactyly [11]. These disorders are characterized by a similar phenotype and overlapping of symptoms, so it is often impossible to make a clear diagnosis.

With regard to diagnosis, CLOVES-specific mutations are not well expressed in blood samples. Thus, genetic sequencing typically needs to require evaluation of at least one tissue sample from affected and unaffected tissues. Chang et al. in 2017 explained that Sanger sequencing may not be efficient for overgrowth syndromes characterized by somatic variants in genes related to PI3K/ AKT/mammalian target of rapamycin (mTOR) [12]. In the same research Chang et al. showed that next-generation sequencing (NGS) technology may help to identify the low-level mosaicism and therefore enable diagnosis of mosaic overgrowth syndromes [12].

Vascular malformations comprise a significant problem that affects patients with CLOVES syndrome. Venot et al. in 2018 reported that targeted treatment with a PIK3CA inhibitor (BYL719) was free of drug-related side effects in 19 patients with complex PROS vascular malformations. The therapy revealed effective vascular responses as well [13]. Currently BYL719 has entered phase I-II clinical trials. Their purpose is to evaluate BYL719 safety and efficacy in patients with various PIK3CA-implanted solid tumours [11]. López Gutiérrez et al. in 2019 [14] reported a 17-year-old girl with CLOVES syndrome, who had massive vascular malformations involving the external genitalia. She was taking oral rapamycin, but with no effect. One month after starting low-dose BYL719 treatment, the patient presented a reduction in the size of the vascular malformations and improved quality of life.

Gustin et al. in 2008 showed that somatic mutations in the PIK3CA gene also occur at high frequency in breast and other cancers [15]. Recent evidence suggests that inhibitors of the phosphoinositide 3 kinase (PI3K) pathway, which are currently being tested in cancer, may provide a treatment option for patients with overgrowth syndromes [16]. Cancer therapeutic drug candidates targeting the PI3K pathway offer hope of much-needed targeted therapies for overgrowth syndromes. However, trials require randomization, blinding, and placebo control to assess their true efficacy.

Taking into account the results of the molecular examination and the clinical picture, our patient was diagnosed with a PROS disorder. However, her symptoms and somatic mutation in the PIK3CA gene (c.1357G>A) revealed in the WES study strongly indicate the diagnosis of CLOVES syndrome.

CLOVES syndrome is extremely rare. In summary, there is no absolute cure for CLOVES syndrome, and all initiatives are targeted at improving the quality of life [12].

\section{ACKNOWLEDGMENTS}

We thank the patient and her family members for their participation in this study.

This work was in part supported by a Statutory Grant of Wroclaw Medical University (SUB.E160.19.004) as well as by the National Science Centre (NCN) Poland grant 2017/25/N/NZ4/00250 to AW. DNA sequencing was 
carried out with the use of CePT infrastructure (Innovative economy 2007-13, Agreement POIG.02.02.00-14024/08-00)

\section{DISCLOSURE}

The authors declare no conflict of interest.

\section{REFERENCES}

1. Bloom J, Upton J $3^{\text {rd }}$. CLOVES syndrome. J Hand Surg Am 2013; 38: 2508-2512.

2. Sapp JC, Turner JT, van de Kamp JM, et al. Newly delineated syndrome of congenital lipomatous overgrowth, vascular malformations, and epidermal nevi (CLOVE syndrome) in seven patients. Am J Med Genet A 2007; 143A: 2944-2958.

3. Alomari AI. CLOVE(S) syndrome: expanding the acronym. Am J Med Genet A 2009; 149A: 294

4. Kurek KC, Luks VL, Ayturk UM, et al. Somatic mosaic activating mutations in PIK3CA cause CLOVES syndrome. Am J Hum Genet 2012; 90: 1108-1115.

5. Nathan N, Keppler-Noreuil KM, Biesecker LG, et al. Mosaic Disorders of the PI3K/PTEN/AKT/TSC/mTORC1 Signaling Pathway. Dermatol Clin 2017; 35: 51-60.

6. Uller W, Fishman SJ, Alomari AI. Overgrowth syndromes with complex vascular anomalies. Semin Pediatr Surg 2014; 23: 208-215.

7. Mirzaa G, Conway R, Graham JM Jr, et al. PIK3CA-Related Segmental Overgrowth. 2013 Aug 15. In: Adam MP, Ardinger HH, Pagon RA, et al. (ed.). GeneReviews ${ }^{\circledast}$ [Internet]. Seattle (WA): University of Washington, Seattle; 1993-2020.

8. Sarici D, Akin MA, Kurtoglu P, et al. A Neonate with CLOVES Syndrome. Case Rep Pediatr 2014; 2014: 845074.

9. Mahajan VK, Gupta M, Chauhan P, et al. Cloves Syndrome: A Rare Disorder of Overgrowth with Unusual Features - An Uncommon Phenotype? Indian Dermatol Online J 2019; 10: 447-452.

10. Alomar S, Khedr RE, Alajlan S. CLOVES Syndrome in a Ninemonth-old Infant. Cureus. 2019; 11: e5772.

11. Keppler-Noreuil KM, Rios JJ, Parker VE, et al. PIK3CA-related overgrowth spectrum (PROS): diagnostic and testing eligibility criteria, differential diagnosis, and evaluation. Am J Med Genet A 2015; 167A: 287-295.

12. Chang F, Liu L, Fang E, et al. Molecular Diagnosis of Mosaic Overgrowth Syndromes Using a Custom-Designed Next-Generation Sequencing Panel. J Mol Diagn 2017; 19: 613-624.

13. Venot Q, Blanc T, Rabia SH, et al. Targeted therapy in patients with PIK3CA-related overgrowth syndrome. Nature 2018; 558: 540-546.

14. López Gutiérrez JC, Lizarraga R, Delgado C, et al. Alpelisib Treatment for Genital Vascular Malformation in a Patient with Congenital Lipomatous Overgrowth, Vascular Malformations, Epidermal Nevi, and Spinal/Skeletal Anomalies and/or Scoliosis (CLOVES) Syndrome. J Pediatr Adolesc Gynecol 2019; 32: 648-650.

15. Gustin JP, Cosgrove DP, Park BH. The PIK3CA gene as a mutated target for cancer therapy. Curr Cancer Drug Targets 2008; 8: 733-740.

16. Madsen RR, Vanhaesebroeck B, Semple RK. Cancer-Associated PIK3CA Mutations in Overgrowth Disorders. Trends Mol Med 2018; 24: 856-870. 